\title{
Reciprocal Circular Polarization Handedness Conversion using Chiral Metamaterials
}

\author{
O. Fernández, Á. Gómez, J. Basterrechea, Member, IEEE, A. Vegas, Member, IEEE
}

\begin{abstract}
Chiral metamaterials are widely used to implement devices that transform the polarization of an incident electromagnetic wave, such as polarization rotators and linear to circular converters, but rarely used as circular polarization converters. This letter presents a chiral metamaterial that changes the handedness of a circularly polarized incident wave. This structure, which presents subwavelength thickness, provides polarization conversion with independence on both the front and back directions and the handedness of the incident wave. High polarization conversion efficiency is shown by both simulations and experiments.
\end{abstract}

Index Terms - Circular polarization, Circular polarization converter, Chiral Metamaterial

\section{INTRODUCTION}

$\mathrm{P}$ OLARIZATION is a characteristic key of electromagnetic (EM) waves in many fields such as wireless communications, liquid crystal display [1], optical data storage [2] or quantum information [3]. Since these applications are polarization sensitive, it is very important to control and manipulate the polarization state of the propagating EM wave.

Polarization rotators and linear to circular polarizers are two traditional devices, comprised by multilayer structures, which transform the polarization state of an incident wave at microwave frequencies. The first one rotates the polarization plane of an incident linearly polarized wave a certain angle without changing its polarization state [4], [5]. The second one converts a linearly polarized wave into a circularly polarized one [6], [7].

Another way to manipulate the polarization is by using chiral media. It is well known that these kind of media present different refractive indices for each of the two circularly polarized eigenwaves. Different real parts of the refractive indices produce different phase delays for the right- and lefthanded circularly polarized eigenwaves and, consequently, a rotation of the polarization plane (optical activity). Meanwhile, different imaginary parts result in different absorption for each eigenwave, causing a transformation on

Manuscript received December 21, 2016. Revision submitted May 10, 2017. Accepted June 12, 2017.

This work has been partially supported by the Spanish Government MINECO through the FEDER co-funded Research Project TEC2014-55463C3-3-P and the Research Project TEC2012-33321. All the authors are with the Departamento de Ingeniería de Comunicaciones, University of Cantabria, Santander, Spain (e-mail: fernanos@unican.es, gomezal@unican.es, basterrj@unican.es, vegasa@unican.es). the polarization conditions (circular dichroism).

Multilayer structures [4]-[7] and bulky chiral media [8] require large thickness, similar or even greater than the operation wavelength. This limitation can be a drawback at certain frequency regimes. Fortunately, with the introduction of metamaterials, there have appeared new structures, as planar chiral metamaterials (CMMs), which provide high optical activity with subwavelength thicknesses. It can be found in the literature several planar CMM based structures printed on PCB that act as polarization rotators [9]-[12] or linear to circular converters [13]-[16].

Besides the aforementioned applications, another less common device that acts over the polarization is the circular polarization converter. This device changes circularly polarized incident waves into the opposite handedness ones. There are few references in the literature that present circular polarization converters implemented on PCB at microwave and optical frequencies [17], [18]. Reference [17] presents a bi-layered planar CMM comprised of asymmetrical split rings that shows circular polarization conversion that depends on both the handedness and the direction of the incident wave.

In this paper, we feature a new CMM based circular polarization converter at microwave regime (X-band). The CMM structure is constituted by short metallic strips orthogonally placed to continuous metallic ones. The proposed circular polarization converter provides high polarization conversion ratio with independence of the incoming circular polarization type. Due to the reciprocity of its geometrical pattern, the response of this metamaterial remains unchanged for the opposite direction of propagation.

The content of the paper is organized as follows. First, Section II details the conditions that the linear and circular transmission coefficients of a structure must fulfil so that it can behave as a circular polarization converter. Following the aforementioned requirements, a novel asymmetric chiral metamaterial structure is introduced. Next, Section III presents simulation and experimental results that show the efficiency of the proposed converter by means of the circular transmission coefficients, the axial ratio and the polarization conversion ratio. Finally, conclusions are drawn in Section IV.

\section{CIRCULAR POLARIZATION CONVERTER}

\section{A. Circular polarization converter characteristics}

This section introduces the conditions that a circular polarization converter must fulfil to transform left- or righthanded circular polarization into its orthogonal one. 
The ideal transmission matrix of the circular polarization converter presents null co-polar transmission coefficients and unitary cross-polar transmission ones, i.e.,

$$
T_{C P}=\left(\begin{array}{ll}
t_{++} & t_{+-} \\
t_{-+} & t_{--}
\end{array}\right)=\left(\begin{array}{ll}
0 & 1 \\
1 & 0
\end{array}\right)
$$

wherein $t_{i j}$ is the transmission coefficient that relates transmitted and incident circular polarized waves with polarization states $i$ and $j$, respectively; the sub-indices $i$ and $j$ refer to right- $(\mathrm{RHCP},+)$ or left-handed $(\mathrm{LHCP},-)$ circular polarization.

It is well known that the electric field of a circularly polarized wave propagating in the $+\mathrm{z}$ direction can be decomposed into the sum of two orthogonal equal-amplitude linearly-polarized electric fields with a phase shift of $\pm 90^{\circ}$

$$
\begin{aligned}
\mathbf{E}_{ \pm}^{\mathrm{inc}} & =E_{x}^{\mathrm{inc}} \hat{a}_{x}+E_{y}^{\mathrm{inc}} \hat{a}_{y}= \\
& =E_{i}\left[\hat{a}_{x} \cos (\omega t-\beta z \pm \pi / 2)+\hat{a}_{y} \cos (\omega t-\beta z)\right] .
\end{aligned}
$$

The incident and transmitted linearly polarized electric field components are related by the transmission matrix for linearly polarized fields

$$
\left(\begin{array}{l}
E_{x}^{\mathrm{tra}} \\
E_{y}^{\mathrm{tra}}
\end{array}\right)=T_{L P}\left(\begin{array}{l}
E_{x}^{\mathrm{inc}} \\
E_{y}^{\mathrm{inc}}
\end{array}\right)=\left(\begin{array}{ll}
t_{x x} & t_{x y} \\
t_{y x} & t_{y y}
\end{array}\right)\left(\begin{array}{l}
E_{x}^{\mathrm{inc}} \\
E_{y}^{\mathrm{inc}}
\end{array}\right) .
$$

Here, $t_{a b}$ represents the transmission coefficient of a linearly polarized wave with polarization type $a$ when impinging with a wave with polarization $b$, where $a$ and $b$ can be $x$ o $y$.

The coefficients of the transmission matrices $T_{C P}$ and $T_{L P}$, (1) and (3), are related by means of the Jones Matrix [19]:

$$
\left(\begin{array}{ll}
t_{++} & t_{+-} \\
t_{-+} & t_{--}
\end{array}\right)=\frac{1}{2}\left(\begin{array}{ll}
t_{x x}+t_{y y}+j\left(t_{y x}-t_{x y}\right) & t_{x x}-t_{y y}+j\left(t_{y x}+t_{x y}\right) \\
t_{x x}-t_{y y}-j\left(t_{y x}+t_{x y}\right) & t_{x x}+t_{y y}-j\left(t_{y x}-t_{x y}\right)
\end{array}\right) .
$$

If the cross-polar transmission coefficients are small enough to be neglected, $\left|t_{y x}\right|,\left|t_{x y}\right| \approx 0$ (condition 1), and the co-polar transmission coefficients have equal amplitudes (condition 2) but are $\pi \mathrm{rad}$ out of phase (condition 3), i.e. $t_{x x} \cdot e^{j \pi}=t_{y y}=t$, Eq. (1) can be simplified as

$$
\left(\begin{array}{ll}
t_{++} & t_{+-} \\
t_{-+} & t_{--}
\end{array}\right)=\frac{1}{2}\left(\begin{array}{cc}
t e^{j \pi}+t & t e^{j \pi}-t \\
t e^{j \pi}-t & t e^{j \pi}+t
\end{array}\right)=-\left(\begin{array}{cc}
0 & t \\
t & 0
\end{array}\right) .
$$

Under this three conditions, the transmitted circular polarized field can then be expressed as

$$
\begin{aligned}
\mathbf{E}^{\mathrm{tra}} & =E_{x}^{\mathrm{tra}} \hat{a}_{x}+E_{y}^{\mathrm{tra}} \hat{a}_{y}=\left[t_{x x} E_{x}^{\mathrm{inc}} \hat{a}_{x}+t_{y y} E_{y}^{\mathrm{inc}} \hat{a}_{y}\right]= \\
& =t\left[\mathrm{e}^{\mathrm{j} \pi} E_{x}^{\mathrm{inc}} \hat{a}_{x}+E_{y}^{\mathrm{inc}} \hat{a}_{y}\right]= \\
& =t E_{i}\left[\hat{a}_{x} \cos (\omega t-\beta z \mp \pi / 2)+\hat{a}_{y} \cos (\omega t-\beta z)\right]
\end{aligned}
$$

where it is shown the change of the circular polarization handedness with respect to the incident one (2).

\section{B. Design of the Composite Chiral metamaterial structure}

The proposed circular polarization converter unit cell is formed by a dielectric slab with a metallic pattern printed on both sides. This design consists of two parallel short strips of length $l_{2}$ interconnected by a continuous metallic wire (Fig. 1a). The distance between the short strips is $l_{l}$. In order to enhance the circular cross-polarization conversion, the structure lacks $\mathrm{C}_{4}$ symmetry. The continuous metallic strips provide high $E_{y}$ transmission meanwhile the combination of short and long strips provides high $E_{x}$ transmission and a $\pi$ rad phase shift. The unit cell presents the same pattern on both faces and, consequently, it is reciprocal for forward and backward propagation.

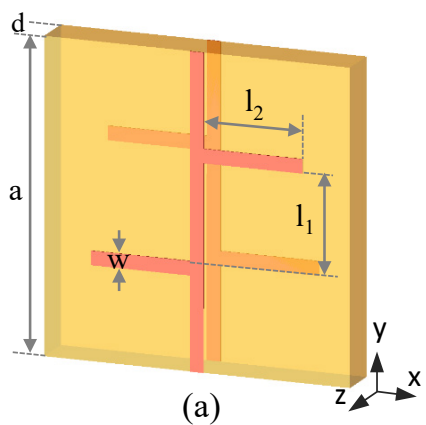

(b)

Fig. 1. (a) Asymmetric CMM unit cell, (b) Detail of the manufactured sample.

The Polarization Conversion Ratio (PCR), defined as $\mathrm{PCR}_{ \pm}=\left|t_{ \pm}\right|^{2} /\left(\left|t_{ \pm}\right|^{2}+\left|t_{\mp}\right|^{2}\right)$, is an indicator of the transformation degree of the transmitted polarization regarding the incident one. Thus, the higher the PCR, the better the polarization conversion is. However, a polarization converter should combine a high PCR and high cross-polar transmission in order to be an efficient converter

\section{RESULTS}

The numerical simulations have been performed using Keysight EMPro ${ }^{\mathrm{TM}}$ 3D EM simulation software. For this purpose, periodic conditions have been applied to the $x$ and $y$ outer boundaries and absorbing conditions to the $z$ outer boundaries.

In our experiment, a CMM sample with $21 \times 21$ unit cells, detailed in Fig. 1b, was fabricated by laser etching technique with a LPKF Protolaser S. The measurements were performed in an anechoic chamber using an Agilent E8362A network analyser with two standard gain X-band horn antennas [20].

Both in measurements and simulations, linearly polarized incident waves are used. For $x$ and $y$ polarizations, the scattering parameters are extracted. From these parameters, the lineal transmission coefficients (3) and the circular ones (4) are then obtained [20].

The geometrical parameters and the substrate characteristics of the proposed Chiral Metamaterial are chosen with the aid of Keysight EMPro ${ }^{\mathrm{TM}}$ and the aim of maximizing the PCR. The chosen pattern parameters are (in $\mathrm{mm}$ ): $a=11, w=0.5, l_{1}=$ 3.4 and $l_{2}=3.55$. The dielectric losses $(\tan \delta)$ have a great impact on the performance of the circular polarization converter. This fact can be seen in Table I, wherein numerical results show that the bigger the value of $\tan \delta$, the lower the cross-polar circular transmission coefficient and the PCR. This degradation is due to the fact that the losses affect in a 
different way to the transmission of $E_{x}$ and $E_{y}$, presenting more influence on $E_{x}$. In order to prevent this drawback, we have selected a low-losses substrate, Rogers RO4003C, characterized by a design dielectric constant $\varepsilon_{r}=3.55$, a dissipation factor $\tan \delta=0.0027$, a thickness $d=1.52 \mathrm{~mm}$ and a copper cladding of $35 \mu \mathrm{m}$.

TABLE I.

NUMERICAL CALCULATIONS OF LINEAR AND CIRCULAR TRANSMISSION COEFFICIENTS, AND PCR FOR DIFFERENT VALUES OF TAN $\delta$

\begin{tabular}{|c|c|c|c|c|c|c|c|c|}
\hline tan $\delta\left(\times \mathbf{1 0}^{-3}\right)$ & $\mathbf{1 . 5}$ & $\mathbf{3 . 5}$ & $\mathbf{5 . 5}$ & $\mathbf{7 . 5}$ & $\mathbf{9 . 5}$ & $\mathbf{1 1 . 5}$ & $\mathbf{1 3 . 5}$ \\
\hline $\boldsymbol{t}_{\boldsymbol{y} \boldsymbol{y}}$ & \multicolumn{7}{|c|}{0.97} \\
\hline $\boldsymbol{t}_{\boldsymbol{x} \boldsymbol{x}}$ & 0.91 & 0.83 & 0.76 & 0.7 & 0.66 & 0.6 & 0.57 \\
\hline $\boldsymbol{t}_{-+}, \boldsymbol{t}_{+-}$ & 0.93 & 0.9 & 0.86 & 0.83 & 0.8 & 0.78 & 0.76 \\
\hline $\boldsymbol{t}_{++}, \boldsymbol{t}_{--}$ & 0.04 & 0.08 & 0.12 & 0.14 & 0.17 & 0.19 & 0.2 \\
\hline PCR & 1 & 1 & 0.99 & 0.98 & 0.97 & 0.96 & 0.94 \\
\hline
\end{tabular}

Fig. 2 presents the transmission coefficients (3) versus frequency for a linearly polarized field obtained by means of numerical simulations and experimental measurements. The measured and simulated results exhibit a similar response but with a frequency displacement of about $40 \mathrm{MHz}$. This frequency shift is mainly due to the manufacturing process that slightly narrow the metallic strips.
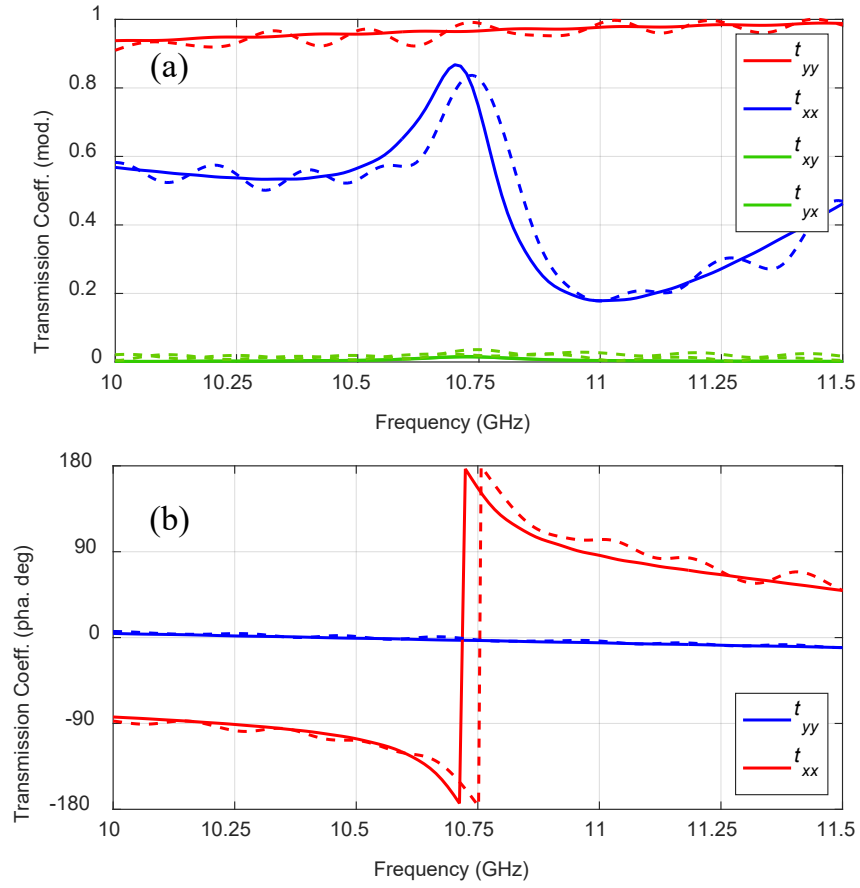

Fig. 2. Linear transmission coefficients obtained from simulation (continuous) and measurements results (dashed). (a) Module (b) Phase of $t_{x x}$ and $t_{y y}$.

From these results, it can be checked that the transmission coefficients fulfil the three design conditions listed in Section $2 \mathrm{~A}$ around the frequency $f_{c}=10.72 \mathrm{GHz}$ in the simulation and at $f_{c}=10.76 \mathrm{GHz}$ in the experimental data. Regarding condition 1, both computed and measured cross-polar transmission coefficients, $t_{x y}$ and $t_{y x}$, present very low amplitudes (see Fig. 2a), below 0.03 in all the frequency band. The maximum co-polar transmission coefficients modules are very similar (Condition 2 ), $\left|t_{x x}\right| \approx 0.89\left|t_{y y}\right|$ in the simulation results and $\left|t_{x x}\right| \approx 0.84\left|t_{y y}\right|$ in the experimental ones (see Fig. 2a). Finally, as stated in condition 3, a difference of $\pi \mathrm{rad}$ between the phases of the co-polar transmission coefficients is obtained at $f_{c}$ (see Fig. $2 \mathrm{~b}$ ).

From $t_{x x}, t_{y y}, t_{y x}$, and $t_{x y}$ the transmission coefficients for circularly polarized waves are calculated using (1) and shown in Fig. 3. This graph shows that the proposed structure works as a circular polarization converter around $f_{c}$, featuring in simulations and measurements high transmission of the crosspolar component, 0.9. The co-polar transmission coefficients are slightly higher in the measured results, due to the finite size of the CMM sample. In both simulated and measured results, $t_{++}$and $t$.- are slightly lower for an incident LHCP wave than for a RHCP wave.

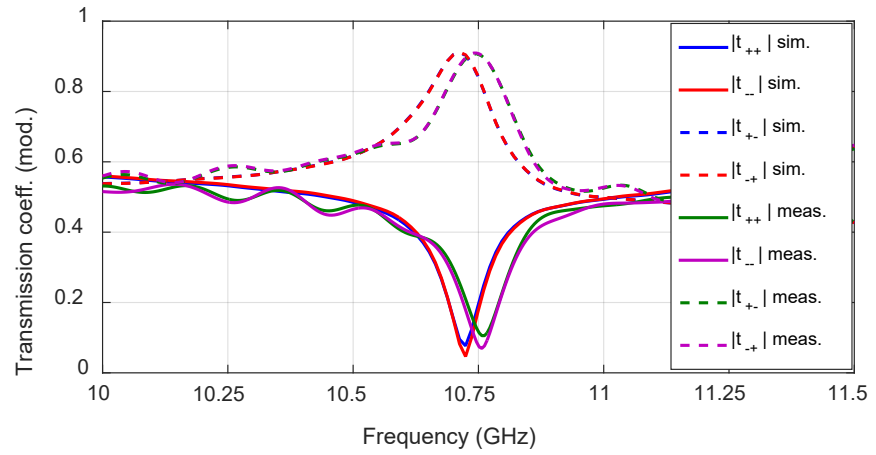

Fig. 3. Simulation and experimental circular transmission coefficients (module)

With these transmission coefficients, the maximum PCR, calculated from (7), is very close to 1 , resulting a PCR $=0.99$ from the simulations and PCR $=0.98$ from the experiments, (Fig. 4). It is noticeable that because $t_{++}>t_{\text {-., }}$, the PCR when converting from LHCP to RHCP is a bit higher than from RHCP to LHCP.

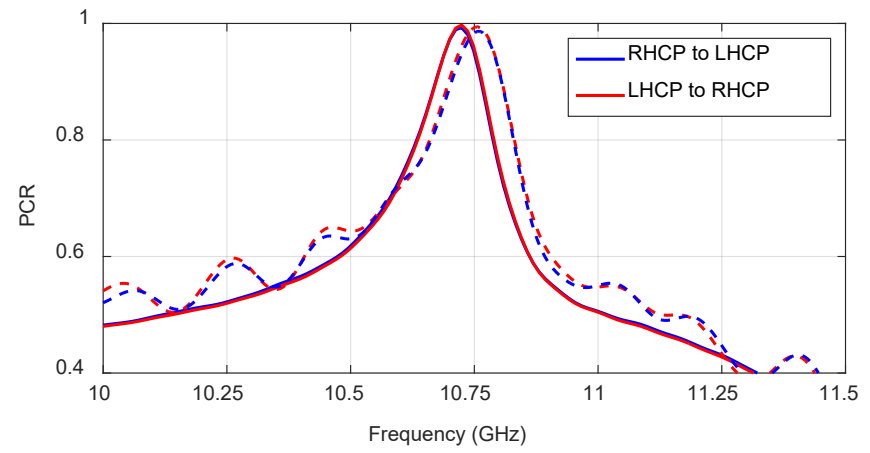

Fig. 4. Simulation (continuous) and experimental (dashed) polarization conversion ratio $(\mathrm{PCR})$

From the above results, it is important to remark that the proposed circular polarization converter presents several improvements in comparison with the circular polarization converter of [17]. On the one hand, it features high cross-polar transmission, $0.9(-0.92 \mathrm{~dB})$, that contrast with the $0.53(-5.6$ $\mathrm{dB})$ of [17]. On the other hand, because of its symmetric design, it exhibits conversion of RHCP and LHCP waves in both front and back propagation directions $(+\mathrm{z}$ and $-\mathrm{z})$ meanwhile [17] only converts one handedness per direction.

To verify the polarization state of the transmitted field, the Axial Ratio (AR) obtained from the measured and simulated 
linear transmission coefficients [21] is displayed in Fig. 5. As shown in Fig. 3, the transmission of the co-polar components is slightly different for LH or RHCP so, for these calculations, we assume circularly polarized incident waves of both handedness $E_{ \pm}^{i n c}= \pm j \hat{a}_{x}+\hat{a}_{y}$. When the incident wave is LHCP, the simulated and measured AR around $f_{c}$ are $0.9 \mathrm{~dB}$ and $1.3 \mathrm{~dB}$, respectively. However, for a RHCP incident wave, the $\mathrm{AR}$ is slightly higher than in the previous case, $1.4 \mathrm{~dB}$ in the simulated results and about $2 \mathrm{~dB}$ in the measured ones.

These values of $\mathrm{AR}$ lower than $3 \mathrm{~dB}$ mean that the polarization converter only changes the handedness of the polarization, independently whether the incident wave is LH or RH, but maintains the circular polarization. The degradation of the AR with respect to $0 \mathrm{~dB}$ is mainly due to the dielectric losses. The results in Table I show that as $\tan \delta$ $i$ increased, one of the linear components, $E_{x}$, is reduced with respect to the other, thus increasing the ellipticity of the polarization. For $\tan \delta \geq 0.0075$ the transmitted wave polarization degenerates on elliptical polarization $(\mathrm{AR}>3$ $\mathrm{dB}$ ). The AR obtained from the measurements is slightly higher than the one calculated through the simulations because of the higher circular co-polar transmission coefficients.

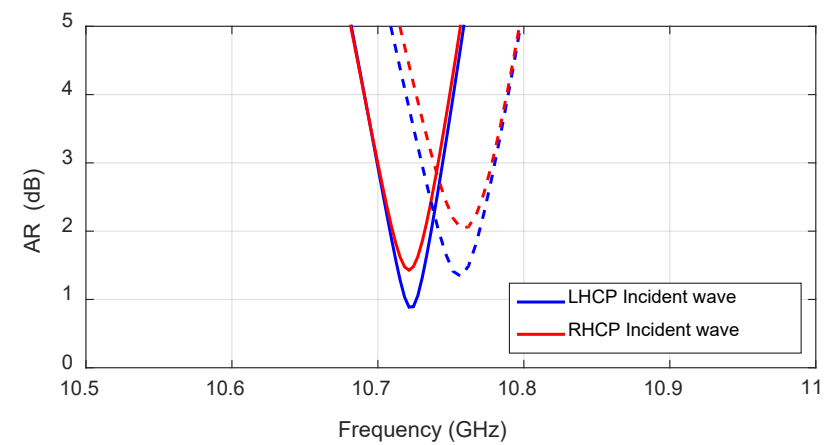

Fig. 5. Axial ratio of the transmitted wave for a LHCP and a RHCP incident wave obtained through simulation (continuous) and experimental (dashed) results.

\section{CONCLUSION}

In this paper it is presented a novel chiral metamaterial structure that behaves as a circular polarization converter, transforming the handedness of an incident circularly polarized wave into its orthogonal one without changing the polarization type. This polarization converter unit cell is formed by the combination of two short metallic strips placed orthogonally to a continuous metallic one. High polarization conversion efficiency with a subwavelength thickness is shown by both simulation and experiment.

Unlike [17], our asymmetric structure provides high polarization conversion ratio and high cross-polar transmission coefficients with independence on both the front and back directions and the handedness of the incident wave.

\section{ACKNOWLEDGMENT}

The authors thank S. Pana, from the University of Cantabria, for her useful work manufacturing the CMM samples and Professor G. Molina-Cuberos, from the
University of Murcia, Spain, for helping with the experimental measurements.

\section{REFERENCES}

[1] J. Lub, P. van de Witte, C. Doornkamp, J. P. A. Vogels and R.T. Wegh, "Stable photopatterned cholesteric layers made by photoisomerization and subsequient photopolymerzation for use as color filters in liquidcrystal displays," Adv. Mater., vol. 15, no. 17, pp. 1420-1425, Sept. 2003.

[2] B. Yao, M. Lei, L. Ren, N. Menke, Y. Wang, T. Fischer and N. Hampp, "Polarization multiplexed write-once-read-many optical data storage in bacteriorhodopsin films," Opt. Lett., vol. 30, no. 22, pp. 3060-3062, Nov. 2005.

[3] A. G. White, D. F. V. James, W. J. Munro and P.G. Kwiat, "Exploring Hilbert space: Accurate characterization of quantum information," Phys. Rev. A, vol. 65, no. 1, 012301, Dec. 2001.

[4] R. P. Torres and M. F. Cátedra, "Analysis and design of a two-octave polarization rotator for microwave frequency," in IEEE Trans. Antennas Propag.., vol. 41, no. 2, pp. 208-213, Feb. 1993.

[5] R. Lech, M. Mazur, and J. Mazur, "Analysis and Design of a Polarizer Rotator System," in IEEE Trans. Antennas Propag.., vol. 56, no. 3, pp. 844 - 847, Mar. 2008.

[6] D. Lerner, "A wave polarization converter for circular polarization," in IEEE Trans. Antennas Propag., vol. 13, no. 1, pp. 3-7, Jan 1965.

[7] L. Young, L. Robinson, and C. Hacking, "Meander-line polarizer," IEEE Trans. Antennas Propag., vol. 21, pp. 376-378, May 1973.

[8] A. Gomez, A. Lakhtakia, J. Margineda, G. J. Molina-Cuberos, M. J. Nuñez, J. A. Saiz-Ipiña, A. Vegas, M. A. Solano, "Full-Wave Hybrid Technique for 3-D Isotropic-Chiral-Material Discontinuities in Rectangular Waveguides: Theory and Experiment," IEEE Trans. Microw. Theory Tech., vol. 56, no.12, pp. 2815-2825, Dec. 2008.

[9] M. Mutlu and E. Ozbay, "A transparent $90^{\circ}$ polarization rotator by combining chirality and electromagnetic wave tunneling," Appl. Phys. Lett., vol. 100, no. 5, pp. 051909, Jan. 2012.

[10] D. Zarifi, M. Soleimani, V. Nayyeri and J. Rashed-Mohassel, "On the Miniaturization of Semiplanar Chiral Metamaterial Structures," in IEEE Trans. on Antennas and Propagat., vol. 60, no. 12, pp. 5768-5776, Dec. 2012.

[11] Y. Ye and S. He, " $90^{\circ}$ polarization rotator using a bilayered chiral metamaterial with giant optical activity," Appl. Phys. Lett., vol. 96, no. 20, pp. 203501, May 2010.

[12] H. Shi, A. Zhang, S. Zheng and Y. Jiang, "A design of dual-band $90^{\circ}$ polarization rotator using twisted electric-field-coupled resonators," Proc. IEEE-MAPE, 2013, pp. 388-390.

[13] Y. Liu, K. Song, Y. Qi, S. Gu and X. Zhao, "Investigation of Circularly Polarized Patch Antenna With Chiral Metamaterial," in IEEE Antennas and Wireless Propagation Letters, vol. 12, pp. 1359-1362, 2013.

[14] X. Ma, C. Huang, W. Pan, B. Zhao, J. Cui and X. Luo, "A Dual Circularly Polarized Horn Antenna in Ku-Band Based on Chiral Metamaterial," in IEEE Trans. on Antennas and Propagat., vol. 62, no. 4, pp. 2307-2311, April 2014.

[15] H. Xu, G. Wang, M. Qi, T. Cai and T. Cui, "Compact dual-band circular polarizer using twisted Hilbert-shaped chiral metamaterial," Opt. Express, vol. 21, no. 21, pp. 24912-24921, Oct. 2013.

[16] Y. Cheng, Y. Nie, Z. Cheng and R. Z. Gong, "Dual-band circular polarizer and linear polarization transformer based on twisted split-ring structure asymmetric chiral metamaterial," Progress In Electromagnetics Research, vol. 145, pp. 263-272, 2014.

[17] L. Wu, Z. Yang, Y. Cheng, R. Gong, M. Zhao, Y. Zheng J. Duan, and X. Yuan, "Circular polarization converters based on bi-layered asymmetrical split ring metamaterials," Appl. Phys. A, vol. 116, no. 2, pp. 643-648, Aug. 2014.

[18] J. Kaschke, L. Blume, M. Thiel, Wu Lin, Z. Yang and M. Wegener, "Circular polarization converters based on coupled pairs of oppositelyhanded gold helices," 2014 Conf. on Lasers and Electro-Optics (CLEO) - Laser Science to Photonic Applications, San Jose, CA, 2014, pp. 1-2.

[19] C. Menzel, C. Rockstuhl and F. Lederer, "Advanced Jones Calculus for the classification of periodic metamaterials," Phys. Rev. A, vol. 82, no. 5, pp. 053811, Nov 2010

[20] E. Plum, "Chirality and Metamaterials," Thesis for the degree of Doctor of Philosophy (PhD), University of Southampton, UK (2010)

[21] C. A. Balanis, "Antenna Theory: Analysis and Design," 3rd ed. Hoboken, NJ: John Wiley, 2005, Chapter 2. 